\title{
A mutation in the cytosolic O-acetylserine (thiol) lyase induces a genome-dependent early leaf death phenotype in Arabidopsis
}

\author{
Reza Shirzadian-Khorramabad ${ }^{+1,4}$, Hai-Chun Jing ${ }^{+1,2}$, Gerja E Everts' ${ }^{1}$, Jos HM Schippers ${ }^{1,5}$, Jacques Hille1 and \\ Paul P Dijkwel*1,3
}

\begin{abstract}
Background: Cysteine is a component in organic compounds including glutathione that have been implicated in the adaptation of plants to stresses. O-acetylserine (thiol) lyase (OAS-TL) catalyses the final step of cysteine biosynthesis. OAS-TL enzyme isoforms are localised in the cytoplasm, the plastids and mitochondria but the contribution of individual OAS-TL isoforms to plant sulphur metabolism has not yet been fully clarified.

Results: The seedling lethal phenotype of the Arabidopsis onset of leaf death3-1 (old3-1) mutant is due to a point mutation in the OAS-A1 gene, encoding the cytosolic OAS-TL. The mutation causes a single amino acid substitution from Gly 162 to Glu162, abolishing old3-1 OAS-TL activity in vitro. The old3-1 mutation segregates as a monogenic semidominant trait when backcrossed to its wild type accession Landsberg erecta (Ler-0) and the Di-2 accession. Consistent with its semi-dominant behaviour, wild type Ler-0 plants transformed with the mutated old3-1 gene, displayed the early leaf death phenotype. However, the old3-1 mutation segregates in an 11:4:1 (wild type: semi-dominant: mutant) ratio when backcrossed to the Colombia-0 and Wassilewskija accessions. Thus, the early leaf death phenotype depends on two semi-dominant loci. The second locus that determines the old3-1 early leaf death phenotype is referred to as odd-ler (for old 3 determinant in the Ler accession) and is located on chromosome 3. The early leaf death phenotype is temperature dependent and is associated with increased expression of defence-response and oxidative-stress marker genes. Independent of the presence of the odd-ler gene, OAS-A1 is involved in maintaining sulphur and thiol levels and is required for resistance against cadmium stress.
\end{abstract}

Conclusions: The cytosolic OAS-TL is involved in maintaining organic sulphur levels. The old3-1 mutation causes genome-dependent and independent phenotypes and uncovers a novel function for the mutated OAS-TL in cell death regulation.

\section{Background}

The biogeochemical sulphur cycle has a major impact on climate and life. The sulphur-containing amino acid cysteine forms the exclusive building block for organic compounds including glutathione that have been implicated in the adaptation of plants to a wide range of biotic and abiotic stresses [1,2]. Cysteine synthesis creates a link between sulphur reduction and amino acid metabolism

* Correspondence: p.dijkwel@massey.ac.nz

1 Molecular Biology of Plants, Groningen Biomolecular Sciences and Biotechnology Institute, University of Groningen, Kerklaan 30, 9751 NN, Haren, The Netherlands

+ Contributed equally

Full list of author information is available at the end of the article and therefore is a point of convergence for nitrogen and sulphur assimilation. Sulphur assimilation starts with the transport of anionic sulphate into plant cells by a family of plasma membrane associated proton/sulphate cotransporter proteins [3]. Through serial enzymatic reactions, sulphate is first converted into sulphide and cysteine biosynthesis is subsequently catalysed by a bienzyme complex called the cysteine synthase complex (CSC). The initial reaction catalyzed by CSC is the formation of O-acetylserine (OAS) from serine and acetyl- CoA by the activity of serine acetyltransferase (SAT) proteins. Subsequently, O-acetylserine(thiol)lyase (OAS-TL) catalyses the incorporation of sulfide into OAS producing

() 2010 Shirzadian-Khorramabad et al; licensee BioMed Central Ltd. This is an Open Access article distributed under the terms of the B.W 1 ed Central Creative Commons Attribution License (http://creativecommons.org/licenses/by/2.0), which permits unrestricted use, distribution, and reproduction in any medium, provided the original work is properly cited. 
cysteine $[4,5]$. SAT requires the presence of excessive amounts of OAS-TL to gain full activity, whereas OASTL activity is low when bound to SAT [6-8]. SAT and OAS-TL enzyme isoforms are distributed within the cell compartments cytoplasm, plastid and mitochondria and the CSC and its subcellular compartmentation have been suggested to play a crucial role in the control of cysteine biosynthesis [9,1]. In Arabidopsis thaliana, five and nine genes encode for SAT- and OAS-TL-like proteins, respectively [9]. The SAT proteins show functional redundancy in vivo and Arabidopsis plants with mutations in any four of the five SAT genes survived although three of the quadruple mutants showed dwarfism [10]. Four genes encoding OAS-TLs are strongly transcribed according to the Genevestigator database [11], the Arabidopsis e-FP browser database [12] and individual studies [13-15]. These genes encode cytoplasmic (OAS-A1), plastidic $(O A S-T L C)$ and mitochondrial $(O A S-T L B$ and CYSC1) isoforms $[15,16]$. OAS-A1 is probably the only functional cytosolic OAS-TL in Arabidopsis and is responsible for a major part of the total OAS-TL activity in the cell $[17,1,18]$. The contribution of each OAS-TL isoform to plant sulphur metabolism has not yet been fully clarified. Knock out of the cytosolic OAS-TL isoform reduces total cellular OAS-TL activity by 44 to $80 \%$, however no apparent phenotypic differences were observed between the mutant and the wild type when grown under non-stressed conditions $[17,18]$. Nevertheless, the antioxidant capacity of the cytosol was perturbed [17]. Cysteine is also found to be the major factor controlling glutathione (GSH) biosynthesis and phytochelatins (PCs) [19-21]. GSH and other secondary organic sulphur compounds are involved in the scavenging of free radicals and hence have been implicated in the adaptation of plants to a wide range of stresses including the detoxification of xenobiotics and the protection against heavy metal toxicity $[22,23,2]$.

Here we report that the lethal old3-1 phenotype is the consequence of a point mutation in the gene encoding the cytosolic OAS-TL. The old3-1 phenotype is associated with elevated expression of defence-response and oxidative stress marker genes. Interestingly, the semidominant phenotype caused by the mutated protein is depending on genomic context resulting in an early onset of leaf death in Ler-0 and Di-2, but not in the Col-0 and Ws- 0 accessions. Since the mutated protein has no OASTL activity in vitro, these data suggest a novel genomedependent function of the mutated cytosolic OAS-TL.

\section{Results}

The old3-1 early leaf death phenotype is a temperaturedependent trait

The onset of leaf death3-1 (old3-1) mutant was isolated from an EMS-mutagenised Arabidopsis Landsberg erecta
(Ler-0) population as a semi-dominant trait [24]. Figure 1a shows that homozygous and heterozygous old3-1 plants suffer from an early leaf death syndrome. The first signs of leaf yellowing occur after 12 days of growth in homozygous old3-1 seedlings. The visible leaf yellowing was associated with a rapid decrease in chlorophyll content and a concurrent increase in ion leakage (Figure 1b), followed by death of the plant after approximately 4 weeks. Plants heterozygous for the old3-1 mutation show premature leaf yellowing but can, in contrast to the homozygous mutants, finish their life cycle. The early leaf death phenotype was found to be a temperature-dependent trait, and growth at $28^{\circ} \mathrm{C}$ completely suppresses the
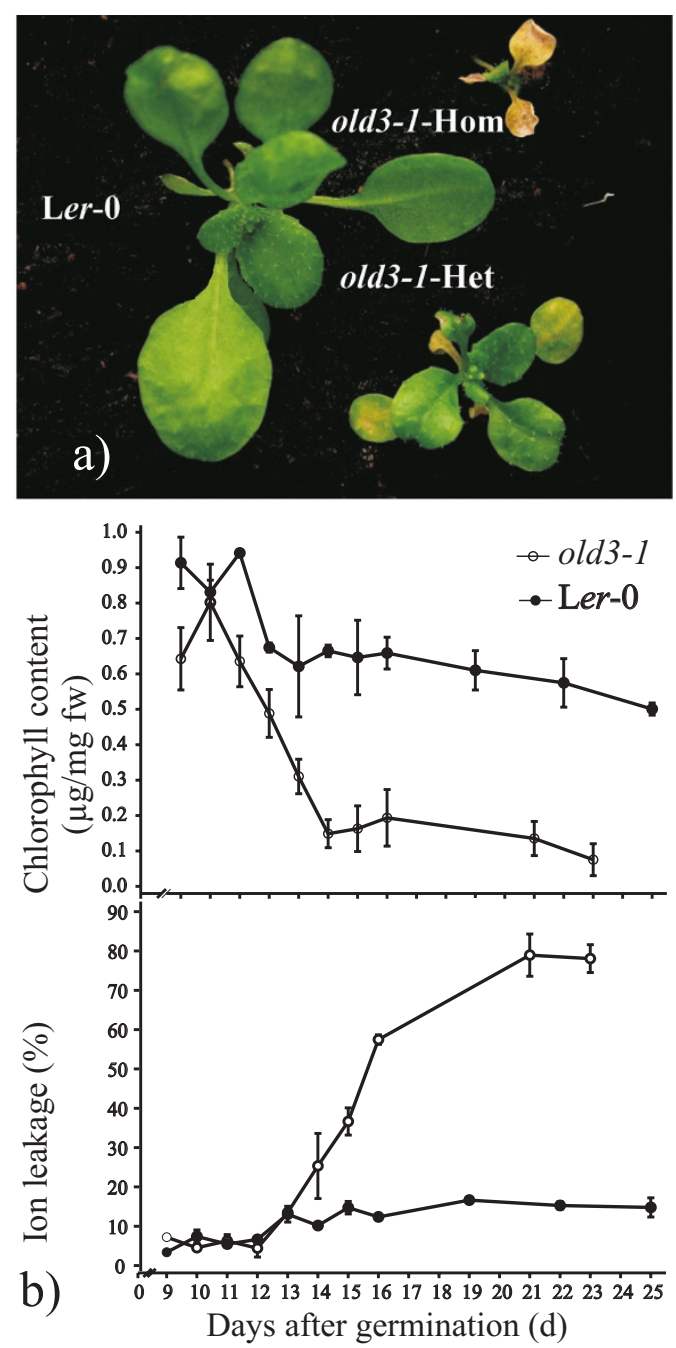

Figure 1 The early leaf death phenotype in old3-1 mutant plants a) Representative 21-day-old soil-grown wild type (Ler-0), old3-1 homozygous and old3-1 heterozygous plants grown in standard conditions at $21^{\circ} \mathrm{C}$. b) Chlorophyll content and ion leakage of cotyledons from Ler-0 and old3-1 homozygous plants grown for up to 25 days. Each data point is shown as mean $\pm \mathrm{sd}$ from 4 replicates. 


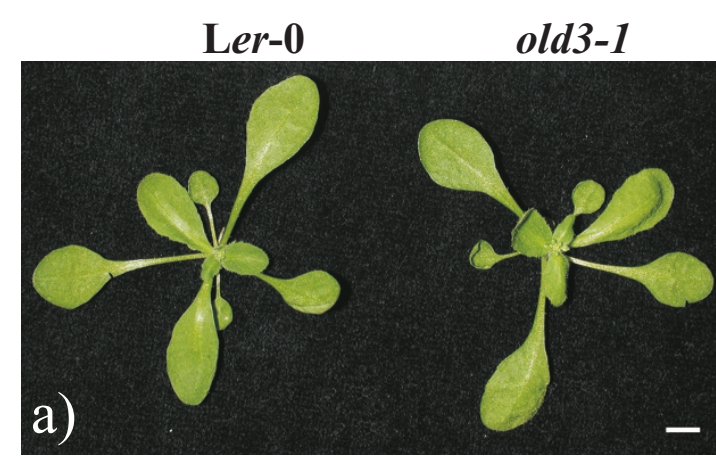

old3-1

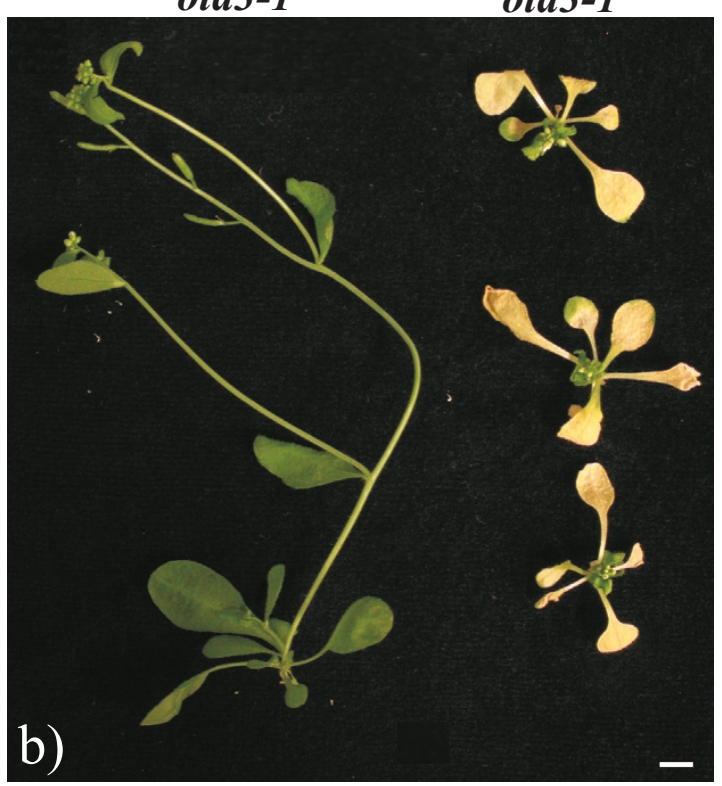

Figure 2 Temperature-dependent phenotypes of old3-1 plants. a) Wild type (Ler-0) and homozygous 21-day-old old3-1 plants grown at $28^{\circ} \mathrm{C}$. b) Representative old3-1 mutants were soil-grown either in 50 $\mu \mathrm{molm}-2 \mathrm{~s}^{-1} \mathrm{Cool}$ white fluorescent light and $28^{\circ} \mathrm{C}$ for 32 days (left) or in $50 \mu \mathrm{molm}^{-2} \mathrm{~s}^{-1} \mathrm{Cool}$ white fluorescent light and $28^{\circ} \mathrm{C}$ for 16 days, and then for another 16 days in $65 \mu \mathrm{molm}-2 \mathrm{~s}^{-1} \mathrm{cool}$ white fluorescent light and $21^{\circ} \mathrm{C}$ (right). Bar represents $0.5 \mathrm{~cm}$.

leaf death phenotype (Figure 2a). Transfer of old3 mutants from $28^{\circ} \mathrm{C}$ to $21^{\circ} \mathrm{C}$ after 2, 4 or 6 weeks results in a visible leaf death phenotype within 10 days (Figure 2b), suggesting that the temperature dependency is independent of the developmental stage. We examined expression of several marker genes involved in developmental senescence, defence response, and programmed cell death in old3-1 and wild type plants. Increased expression of PR1, SAG14, SAG21 and SAG13 was detected in 16- and 24-day-old old3-1 plants, while no signs of $S A G 12$ expression were found [24]. Marker genes of $P R-1$ and $S A G 13$ are associated with programmed cell death as well as oxidative stress signals [25]. Therefore, the expres- sion of a general oxidative stress marker gene DEFL (AT2G43510) [26] was monitored in 12-day-old old3-1 plants, and found to be 160 -fold up-regulated as compared to wild type plants (Figure 3). The results shown here, together with those published previously [24] suggest that early leaf death in old3-1 seedlings does not result from activation of a developmental senescence program, but rather a cell death pathway that is related to the plant immune system and stress responses.

\section{The old3-1 phenotype is a genome-dependent trait in Arabidopsis thaliana}

$\mathrm{F}_{2}$ progeny obtained from a cross between heterozygous old3-1 and Ler-0 plants segregates as a monogenic trait for the old3-1 phenotype, while the same phenotype segregates in a different ratio when crossed to Col-0 [24] (Table 1). Whereas in Ler-0 the old3 mutation is caused by a single gene defect, two genes are needed for the same phenotype in Col-0. The second gene was designated as old3-1 determinant (odd). The Ler-0 and Col-0 alleles were called odd-ler and odd-col, respectively. Different segregation ratios were tested based on the number of plants with a homozygous, a heterozygous and wild type phenotype. The 1:2:13, 1:8:7 and 3:7:8 (homozygous: heterozygous: wild type) segregation ratios were dismissed because of chi-square values of higher than 50 . Of the other segregation ratios tested, only the 1:4:11 ratio fitted the data (Table 1). Thus two old3-1 and two odd-ler alleles need to be present for the homozygous leaf death phenotype to be manifested. The heterozygous phenotype requires either one old3-1 and two odd-ler alleles or one odd-ler and two old3-1 alleles (Table 2). The presence of the odd-ler allele in old3-1 plants, rather than the absence of the odd-col allele, is required for the manifestation of the old3-1 phenotype, thus the odd-ler allele is

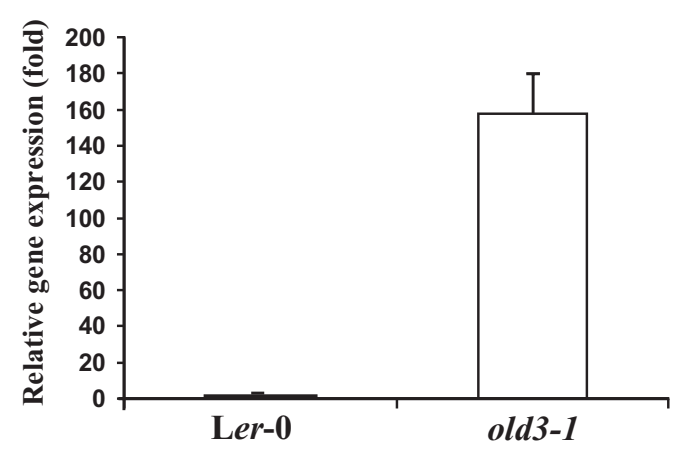

Figure 3 Relative transcript abundance of the DEFL gene. Relative transcript levels of the general oxidative stress-related marker gene DEFL in 12-day-old old3-1 and Ler-0 plants were measured by quantitative Real-Time PCR, using ACTIN2 as internal control. The values shown are the means of three repeats \pm sd. 
Table 1: Phenotypes and segregation of $F_{1}$ and $F_{2}$ progeny of crosses between heterozygous old3-1 plants and various wild type Arabidopsis accessions.

\begin{tabular}{|c|c|c|c|c|c|c|c|c|}
\hline \multirow[b]{2}{*}{ Male } & \multirow[b]{2}{*}{ Female } & \multicolumn{2}{|c|}{ F1 phenotype } & \multicolumn{5}{|c|}{ F2 phenotype and segregation 1} \\
\hline & & Wild type & old3-1 Het & Wild type & old3-1 Het & old3-1 Hom & $x^{2}$ & Segregation \\
\hline old3-1 & Ler-0 & 13 & 12 & 115 & 241 & 123 & 0.197 & $1: 2: 1$ \\
\hline Het $^{1}$ & Di-2 & 29 & 24 & 183 & 382 & 166 & 0.74 & $1: 2: 1$ \\
\hline old3-1 & Col-0 & 49 & 0 & 237 & 88 & 19 & 0.337 & 1:4:11 \\
\hline Het $^{1}$ & Ws-0 & 53 & 0 & 341 & 119 & 32 & 0.18 & 1:4:11 \\
\hline
\end{tabular}

${ }^{1}$ In the crosses between Het-old3-1 and Ler-0, Di-2 accessions, only the $\mathrm{F}_{1}$ progeny with the Het-old3-1 phenotypes were selected and used for $F_{2}$ segregation analyses. For the crosses between Het-old3-1 and Col- 0 and Ws-0, at least ten $F_{1}$ plants were selected and allowed to set seed. The phenotypes of the $F_{2}$ populations were observed and only those in which the old3-1 phenotype segregated were used.

semi-dominant as well. The old3-1 heterozygous plants were crossed to two additional accessions. The old3-1 phenotype segregated in Di-2 as in Ler-0, while the Ws-0 accession behaved similar to Col-0 (Table 1). Thus, different Arabidopsis backgrounds resulted in two distinct segregation patterns for the old3-1 phenotype. The old3-1 mutant was crossed to a further 14 different accessions and the $\mathrm{F}_{1}$ of 11 of those (Ak-1, Bd-0, Bla-2, Bs-2, Litva, Mt-0, Nok-0, Rubezh, Noe-1, Tsu-1, Wil 2) looked like the $F_{1}$ of the cross with Col- 0 . The $F_{1}$ of the other 3 crosses had the old3-1 heterozygous phenotype (Bu-18, Wa-1, Rsch-0), suggesting the presence of odd-ler-like alleles in these accessions.

Initial mapping revealed that the old3-1 phenotype is linked to a CAPS marker locus called G4539a on chromosome 4, and a SSLP marker locus called K11J14 on chromosome 3 [24]. Therefore, one of the genome loca-

Table 2: Genotypes and corresponding phenotypes of old3 and odd allele combinations

\begin{tabular}{ll}
\hline Genotype1 & Phenotype \\
\hline OLD3OLD3odd-colodd-col & Wild type \\
OLD3old3-1odd-colodd-col & Wild type \\
old3-1old3-1odd-colodd-col & Wild type \\
OLD3OLD3odd-lerodd-col & Wild type \\
OLD3old3-1odd-lerodd-col & Wild type \\
old3-1old3-1odd-lerodd-col & Het-old3-1 \\
OLD3OLD3odd-lerodd-ler & Wild type \\
OLD3old3-1odd-lerodd-ler & Het-old3-1 \\
old3-1 old3-1odd-lerodd-ler & Hom-old3-1
\end{tabular}

${ }^{1}$ Genotoypes were determined using DNA markers surrounding the odd region and a marker that distinguishes between the OLD3 and old3-1 alleles. tions carries the old3-1 mutation, and the other the oddler gene. To clarify the position of the old3-1 and odd-ler loci on the genome, plants heterozygous for the old3-1 mutation were crossed to $f c a-1$ and abi1-1 mutants (both having the Ler-0 background). The old3-1 mutation was found to co-segregate with the $A B I 1$ and $F C A$ wild type alleles (Data not shown), demonstrating that the old3-1 mutation is located on chromosome 4 and the odd-ler gene on chromosome 3 . Thus, the old $3-1$ early leaf death phenotype is a genome-dependent trait in Arabidopsis which requires accession-specific odd-ler-like alleles.

\section{The OLD3 gene encodes the cytosolic 0 -acetylserine (thiol)} lyase, OAS-A1

Further mapping placed the old3-1 mutation into a 14-kb region on the bacterial artificial chromosome clone FCA2 (accession number Z97337) spanning 8 open reading frames. Sequence analysis revealed a $\mathrm{G}$ to A substitution in AT4G14880 which encodes the cytosolic $O$-acetylserine (thiol) lyase (OAS-TL), OAS-A1. The mutation results in a Gly ${ }^{162}$ to Glu ${ }^{162}$ substitution in the OAS-A1/ OLD3 protein. The entire old3-1 gene including its promoter region was cloned and transformed into wild-type Ler-0 plants. Transformation of a single old3-1 gene into Ler-0 is expected to give the old3-1-het phenotype. However, because of possible position effects and multiple integrations, a range of expression levels and phenotypes were expected. More then 20 transformants were identified and 15 transformants were tested for the presence of the basta resistance gene, the transgenic old3-1 gene and the wild type OLD3 gene. All the tested plants had the expected genotype and showed an early leaf death phenotype. However, none of the transformants produced progeny although some phenotypic variation was found with several transformants dying at the two leaf stage similar to the homozygous mutant and others developing up to the flowering stage. Figure 4a shows a photograph 
of a representative old3-1 transformant. Thus, the old3-1 mutation results in a mutant protein with an altered or partial function that causes the early leaf death phenotype.

OAS-A1/OLD3 uses pyridoxal 5'-phosphate (PLP) as a cofactor to synthesise cysteine from $\mathrm{O}$-acetylserine (OAS) and sulfide $[4,5,27]$. According to the amino acid sequence alignment of three OAS-TL isomers in Arabidopsis, the old3-1 amino acid substitution is located at the fifth $\alpha$-helix in the middle of a highly conserved area (TTGPEIW) (Figure 4b) [28]. The fifth $\alpha$-helix, including His ${ }^{157}$ that interacts with the pyridoxal 5'-phosphate (PLP) binding site, is in close proximity to a mostly conserved region at the sixth $\alpha$-helix (GAGKYLK) including Ala $^{189}$, Tyr ${ }^{192}$ and Leu ${ }^{193}$ (Figure 4c). The Gly ${ }^{162}$ to Glu ${ }^{162}$ substitution results in a size and charge difference that may cause a change in the protein structure and activity.

\section{The old3-1 protein has no in vitro OAS-TL activity}

The E.coli Cys- auxotrophic strain NK3 [29] lacks the cysteine synthase gene and consequently is unable to grow on medium without supplemental cysteine. The OAS-A1/OLD3 is able to complement the E. coli NK3 strain [14] and we determined whether the old3-1 gene is able to complement the phenotype of the E.coli NK3 strain as well. The old3-1 and the wild type OAS-A1/ $O L D 3$ cDNAs were cloned into an expression vector. Subsequently, the E. coli NK3 strain was transformed with the expression vectors harbouring the cDNAs and the empty vector and grown on minimal medium M9 with and without cysteine. E. coli NK3 transformed with the $O A S-A 1 / O L D 3$ cDNA could grow on medium lacking cysteine, while E.coli NK3 carrying the old3-1 cDNA and empty vector were unable to grow further (Figure 5). These results confirm that the OAS-A1/OLD3 is able to complement the phenotype of the $E$. coli $\Delta$ cys NK3 strain and suggest that the cytosolic old3-1 protein has either a reduced or no OAS-TL enzyme activity in E.coli NK3.

For further old3-1 OAS-TL activity analysis, the $O A S$ A1/OLD3 and old3-1 cDNAs were cloned into the pET15b expression vector. Subsequently the old3-1 and OAS-A1/OLD3 proteins were over-expressed in E.coli BL21 (DE3). The proteins were purified using a $\mathrm{Ni}^{2+}$ NTA agarose column, and molecular masses on a SDSPAGE gel were found to be in agreement with the expected molecular mass. We applied $3.1 \mathrm{nM}$ purified OAS-A1/OLD3 and old3-1 OAS-TL proteins to the cysteine precursors $\mathrm{OAS}$ and $\mathrm{Na}_{2} \mathrm{~S}$ and subsequently OAS-TL activity was measured according to [30]. A specific activity of $84 \pm 4 \mu \mathrm{mol} \mathrm{min} \mathrm{mg}^{-1} \mathrm{mg}^{-1}\left(48 \pm 2 \mathrm{~s}^{-1}\right)$ was found for the OAS-A1/OLD3, but for the same enzyme concentration no activity was detected for old3-1 OASTL. The obtained value for the OAS-A1/OLD3 protein is within those already published $\left(906 \pm 39 \mu \mathrm{mol} \mathrm{min}^{-1} \mathrm{mg}^{-1}\right.$

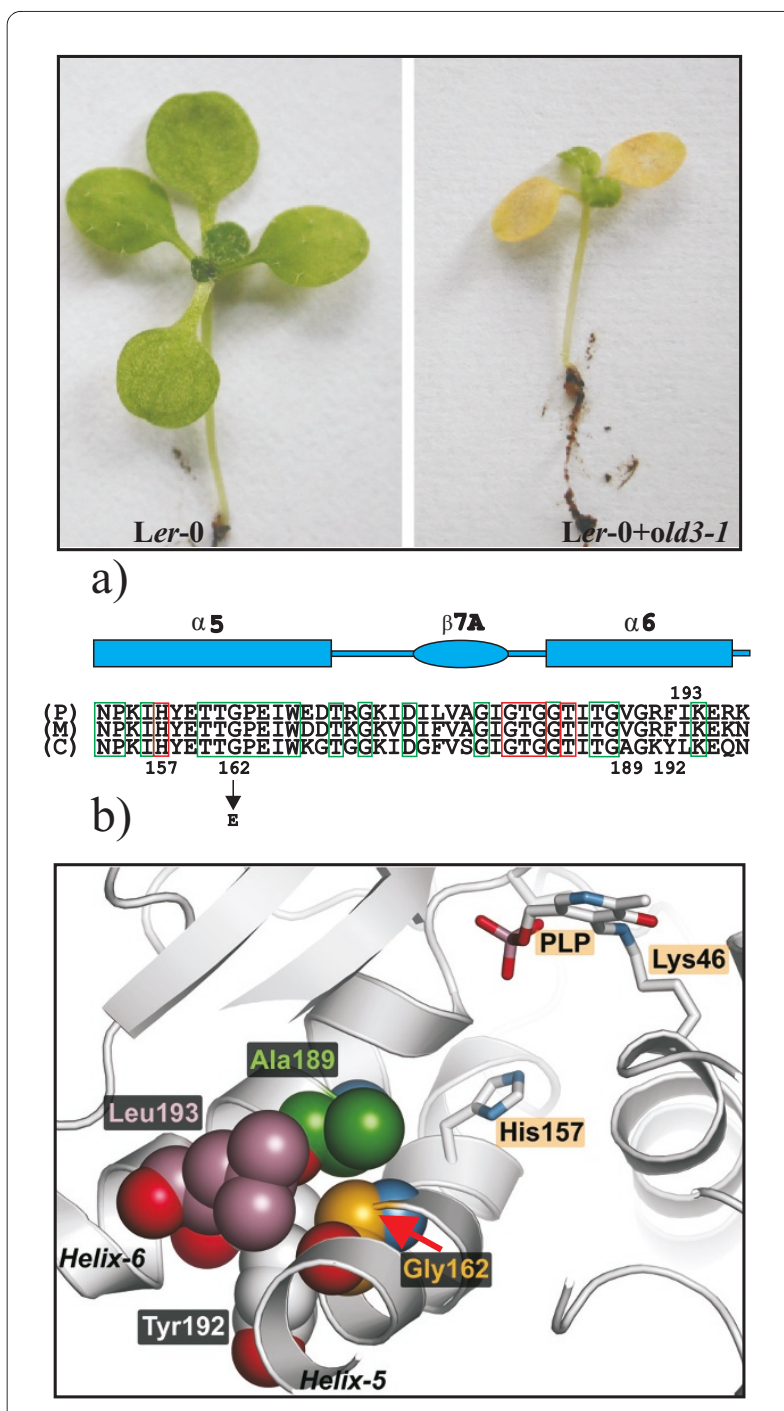

c)

Figure 4 The OLD3 gene encodes the cytosolic OAS-TL. a) The old3-1 genomic region was cloned and transformed into Ler-0 wild type plants. A 14-day-old wild type Ler-0 plant and a representative Ler0 plant carrying the old3-1 transgene are shown. b) Amino acid sequence alignment of the area surrounding $\mathrm{G}^{162}$ of three Arabidopsis OAS-TL proteins. The position of the $\mathrm{G}^{162}$ to $\mathrm{E}^{162}$ substitution in the cytosolic OAS-TL is indicated. Amino acids shown in green boxes are highly conserved amino acids; amino acid residues shown in red boxes are involved in the interaction with pyridoxal 5'-phosphate (PLP). The schematic drawing indicates the location of amino acids in helix-5 and helix-6. P (AT2G43750 - plastid OAS-TL); M (AT2G59760 - mitochondrial OAS-TL); C (AT4G14880-OAS-A1).C) The location of PLP, Lys ${ }^{46}$, which is the only residue forming a covalent bond (the Schiff base) with cofactor PLP, and His ${ }^{157}$ that is involved in interacting with PLP are shown by stick drawings (highlighted black on orange). The position of Gly ${ }^{162}$ (gold) in helix-5 is shown as a CPK model. This residue is in close proximity with residues in helix-6 including Ala ${ }^{189}$ (green), Tyr ${ }^{192}$ (white), and Leu ${ }^{193}$ (purple). Figure made using Pymol, http://www.pymol.org/. 


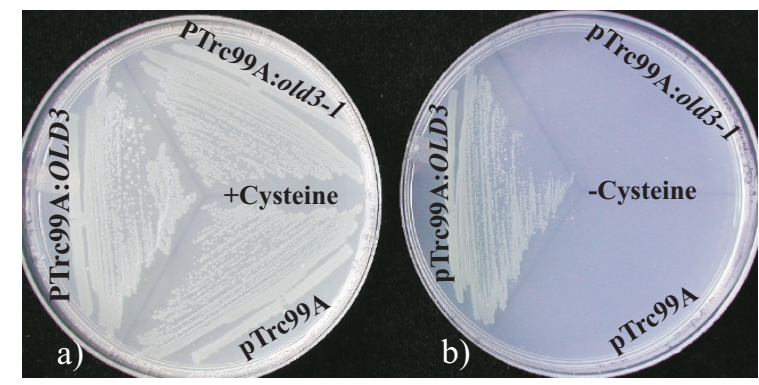

Figure $\mathbf{5}$ Genetic complementation of the $\Delta$ Cys E.coli strain NK3 The empty vector (pTrc99A), and vector harbouring the wild type OASA1 (pTrc99A:OLD3) and mutated old3-1 OAS-TL (pTrc99A:old3-1) cDNAs were transformed into the $\triangle$ Cys E.coli NK3 strain and plated onto M9 medium with (a) or without (b) $0.5 \mathrm{mM}$ cysteine and incubated at $37^{\circ} \mathrm{C}$.

[6], $2 \mu \mathrm{mol} \mathrm{min} \mathrm{mg}^{-1} \mathrm{mg}^{-1}$ [31], and $225 \mu \mathrm{mol} \mathrm{min}^{-1} \mathrm{mg}^{-1}$ [32]). Together, the data show that old3-1 cytosolic OAS-TL has no in vitro activity at the enzyme concentrations tested.

\section{OAS-A1/OLD3 affects sulphur balance and thiol levels}

Under sulphur-sufficient conditions, metabolites such as cysteine and glutathione act as regulators of sulphur uptake and assimilation [33]. In order to clarify if the mutated cytosolic old3-1 OAS-TL has some effects on availability of down-stream or up-stream compounds in the cysteine biosynthesis pathway, we measured the contents of total sulphur and sulphate ion as direct measurements of plant sulphur balance. Total thiol levels were measured as a parameter of plant sulphur metabolism $[34,21,35]$. To be able to distinguish between direct effects of the mutated OASTL and indirect effects as a result of the early leaf death phenotype, we isolated the old3-2 mutant as described in the Methods section. This mutant carries a homozygous T-DNA insertion in the $O A S-A 1 / O L D 3$ gene and is identical to the oasa1.1 mutant as described by Lopez-Martin et al. [17] (results not shown). The old3-2/oas-a1.1 mutant has the Col-0 (odd-col) background and similar to the old3-1old3-1oddcolodd-col line (carrying the homozygous old3-1 mutation and odd-col alleles in an otherwise Col-0/Ler-0 mixed background), did not show the early leaf death phenotype (Figure 6). Thus, the lack of OAS-TL activity in the old3-2/oas-a1.1 mutants does not cause a leaf death phenotype. Figures $7 \mathrm{a}$ and $7 \mathrm{~b}$ show the total sulphur and sulphate ion contents as well as thiol levels of the old3 mutant and their respective wild types. Total sulphur and sulphate ion content was increased while the thiol content was lower in old3-1 and old3-2/oasa1.1 lines as compared to their wild types. The data show that the $O A S-A 1 / O L D 3$, independently from the odd-ler gene affects sulphur balance as well as thiol levels.

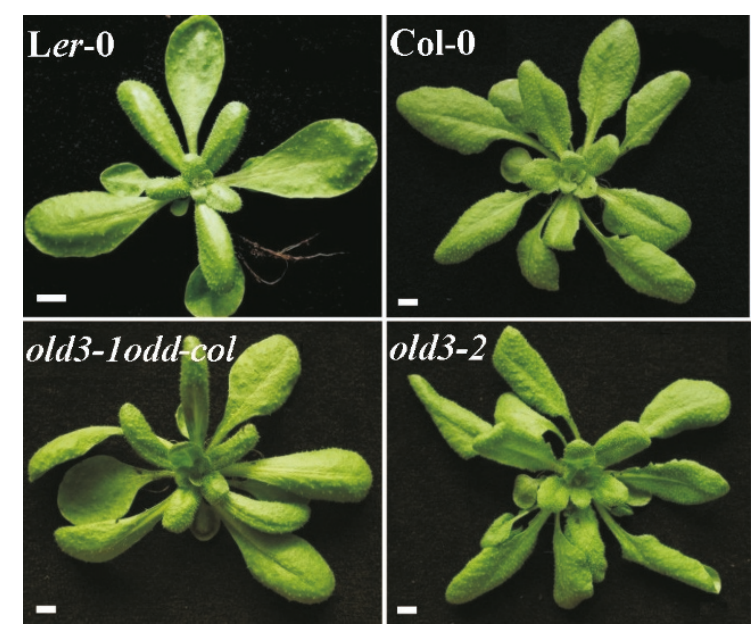

Figure 6 Phenotypes of wild type and old 3 mutant plants. Representative 27-d-old soil-grown plants of Ler-0, Col-0, old3-1old3-1oddcol odd-col (old3-1odd-col, carrying the homozygous old3-1 mutation and homozygous odd-colalleles in an otherwise Col-0/Ler-0 mixed background) and old3-2/oas-a1.1 (old3-2, carrying a homozygous TDNA insertion in the OLD3 gene) grown in $250 \mu \mathrm{molm}^{-2} \mathrm{~s}^{-1} \mathrm{cool}$ white fluorescent light at $21^{\circ} \mathrm{C}$. Bars represent $0.5 \mathrm{~cm}$.

old3 mutant lines show an increased sensitivity to cadmium stress

$O A S-A 1 / O L D 3$ gene expression is up-regulated in response to cadmium-induced stress and this may allow for enhanced cadmium tolerance [36]. The effect of cadmium on seedling growth of the old3-1 and old3-2/ oasa1.1 mutants and their wild types were investigated. Figure 7c shows the effect of various cadmium concentrations on plant growth. At $150 \mu \mathrm{M}$ cadmium, all tested genotypes showed a considerable decrease in fresh weight as compared to growth without cadmium. In contrast, the old3-1 and old3-2/oas-a1.1 lines showed significant reductions in fresh weight already from $50 \mu \mathrm{M}$ cadmium and onwards. Thus, OAS-A1/OLD3 is involved in cadmium tolerance and the old3-1 mutation increases the cadmium sensitivity, independent of the presence of the odd-ler or odd-col gene.

\section{Discussion}

Cysteine is synthesised in the cytosol, the plastids and mitochondria. The Arabidopsis genome contains OASTL isoforms for each of the different cellular locations. Recently the effects of T-DNA insertions in the genes encoding the different isoforms were studied [18]. Although the cytosolic OAS-A1/OLD3 isoform contributes to $44 \%$ of total OAS-TL activity in leaf and $~ 80 \%$ in root, it was reported that the OAS-A1/OLD3 isoform is completely dispensable [18]. However, others found that loss-of function cytosolic OAS-TL mutants resulted in compromised antioxidant capacity of the cytosol [17]. 


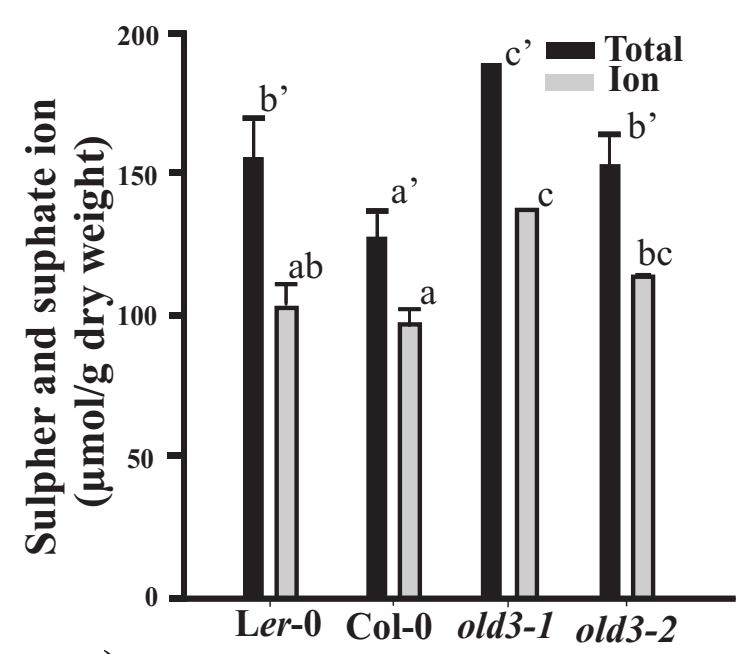

a)
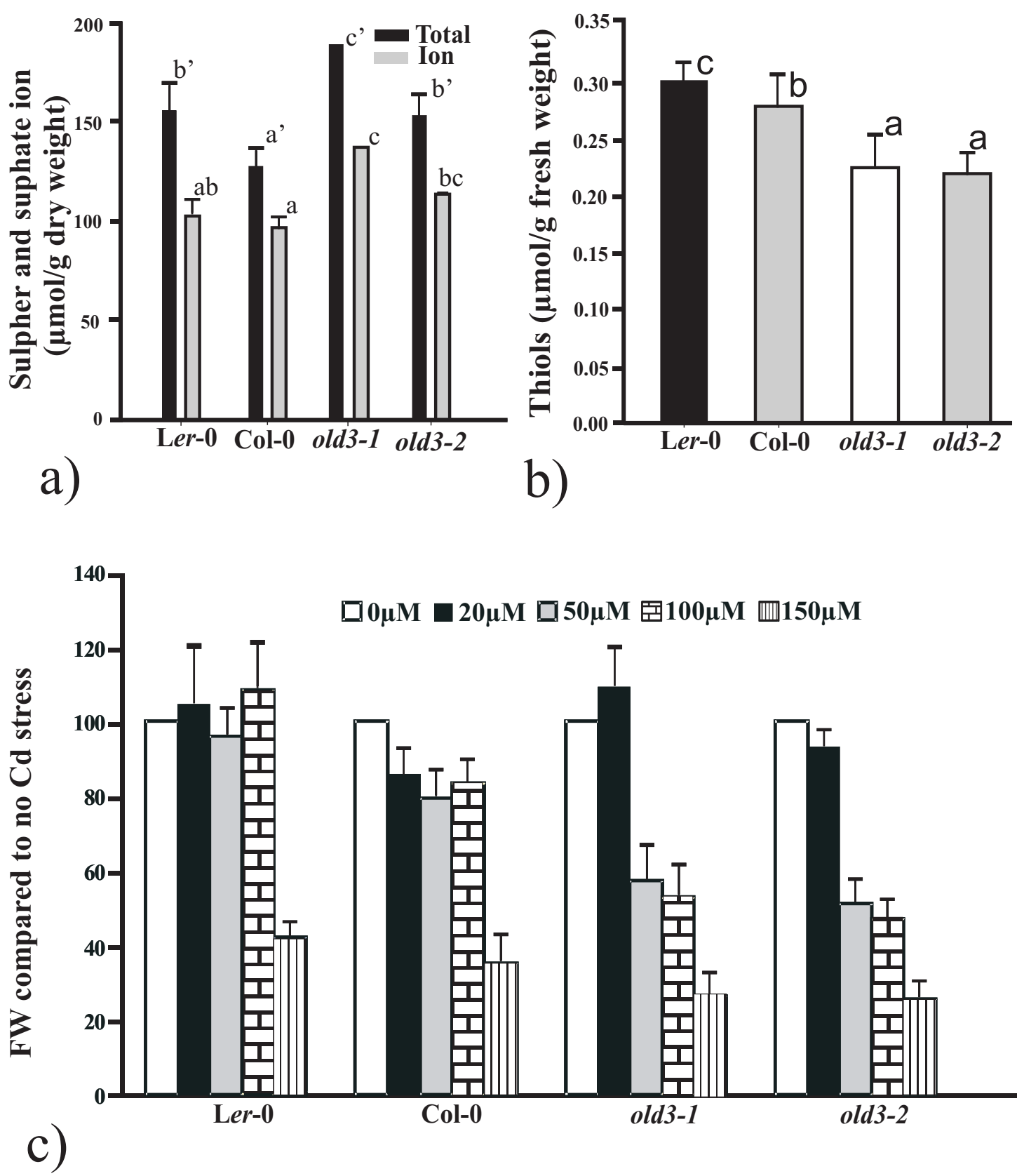

Figure 7 The function of OAS-A1 on sulphur metabolism and cadmium stress. a) Total sulphur and sulphate ion levels were measured in dry matter obtained from plants grown for 27 days in soil saturated with $25 \%$ Hoagland solution. b) Total water-soluble non-protein sulfhydryl (thiol) compounds were measured in fresh materials obtained from 27-day-old plants. Above-ground parts of 27-day-old plants were harvested for measurement of total sulphur, sulphate ion and thiols content. Data are shown as mean \pm sd of four replicates (17 plants for each replicate). Bars with the same letters indicate values that are not significantly different from each other after Duncan multiple variant significance test at a significance level of $\mathrm{p}<0.05$. c) Effect of $\mathrm{CdCl}_{2}$ on fresh weight of old3-1, old3-2 and their respective wild types. Fresh weight was measured after 16 days of growth on the indicated concentration of $\mathrm{CdCl}_{2}$. Fresh weight is expressed as percentage of the fresh weight when grown without $\mathrm{CdCl}_{2}$ (set at 100). Data are shown as mean \pm sd of three replicates (20 seedlings for each replicate).

Here we report that a single amino-acid change in OASA1/OLD3 compromises OAS-TL activity and uncovers a novel role of this enzyme in cell death signalling.

The semi-dominant old3-1 mutation causes a Gly 162 to Glu ${ }^{162}$ substitution in OAS-A1/OLD3. The old3-1 muta- tion results in a dysfunctional enzyme in vitro and likely in vivo as well. The 3D structure of the OAS-A1/OLD3 has been resolved [28] and this allows for an explanation for the compromised enzyme activity: the small and neutral Gly residue is substituted with a much larger charged 
amino acid Glu that is in close proximity to the sixth $\alpha$ helix, therefore likely causing a positional movement in the $\alpha$-helices 5 and 6 , and subsequent shifts of the associated residues in these helices. Consequently, residues involved in the positioning of the substrate and the pyridoxal 5'-phosphate (PLP) cofactor, which is $\sim 16$ A away from Gly ${ }^{162}$, might be affected by these helical shifts $[37,28]$.

The old3-1 early leaf death phenotype is a result of the activity of the mutated old3-1 allele, rather than the absence of the wild type OAS-A1/OLD3 allele, in combination with a natural variant gene odd-ler. The phenotype therefore depends on the genomic context. The odd-lerlike alleles are present in the minority of accessions tested and the presence of odd-col/odd-ler alleles does not correlate with the geographic origins of the accessions, or with their phylogeny relationships as illustrated with molecular markers [38]. It is unclear how the mutated OAS-TL may cause cell death in combination with the odd-ler gene. The CSC formation and protein-protein interaction of its subunits play essential regulatory roles in plants [1] and the old3-1 mutant protein may affect the formation of the CSC and SAT activity. A changed OASTL conformation could exert a dominant-negative effect in combination with the odd-ler gene product. The leaf death phenotype moreover strictly depends on temperature and can conveniently be switched on and off by changing the growth conditions. It seems possible that a higher temperature may alter the folding of the mutated protein or abolish the interaction with the odd-ler gene product or a protein that is involved in the leaf death phenotype. Formally it cannot be excluded that the absence of cytosolic OAS-TL activity causes the early leaf death phenotype in combination with the odd-ler allele. In this scenario, the early leaf death phenotype observed in the Ler-0 plants transformed with the old3-1 gene would have to be a result of co-suppression of the wild type $O L D 3$ gene by the transgene. However, since the obtained transformants all had the early leaf death phenotype and no wild type transformants were found, this possibility seems unlikely.

The old3-2 mutant used in this study is identical to the oas-a1.1 mutation and is likely a knock-out allele [17]. Similar to what has been found earlier $[17,18]$, the old3-2/ oas-a1.1 mutation or the old3-1 mutation in combination with the homozygous odd-col allele (old3-1old3-1oddcolodd-col) resulted in plants that were indistinguishable from the wild type, when grown under standard conditions. Therefore, the compromised activity of OAS-A1/ OLD3 in old3-2 plants does not induce the cell death phenotype. However, a significant increase in both total sulphur and sulphate ion was found in the old3-2/oas-a1.1 mutant. Similar differences were not found by Heeg et al. [18], perhaps due to differences in growth conditions and/or plant age. In addition, a decrease in non-protein thiol contents was found in the old3-1 and old3-2/oasa1.1 mutant lines. GSH is a non-protein thiol and others [17] found a reduction in GSH levels in old3-2/oas-a1.1 plants. These results suggest that the compromised OASA1/OLD3 activity in the mutant causes reduced sulphate assimilation and lower organic sulphur levels. However, the changes are small and may have little, if any, impact on plant growth when grown under standard growth conditions $[18,17]$.

Plants have developed defence systems to cope with environmental stresses such as heavy metal pollution. Cysteine is required for the synthesis of GSH and is as such involved in plant responses to toxic levels of heavy metals [21]. OAS-A1/OLD3 is known to be involved in the defence response of Arabidopsis against abiotic stresses such as salinity and the presence of heavy metals $[17,39,40]$. Moreover, its higher expression resulted in increased cadmium tolerance $[39,36]$. Thus, increased OAS-A1/OLD3 activity enhances cadmium resistance. Here we show that plants with compromised OAS-A1/ OLD3 function are more sensitive to cadmium stress. Consequently, the OAS-A1/OLD3 is required for coping with cadmium-induced stress and the mitochondrial and/or the plastidic OAS-TL cannot fully compensate for the lack of OAS-A1/OLD3. The function of the OAS-A1/ OLD3 may therefore be to quickly respond to environmental stress, while its function during growth under environmentally ideal conditions may be limited. Consistent with our results, old3-2/oas-a1.1 seedlings exhibited an enhanced sensitivity to cadmium-induced stress [17]. In addition, the data from transcriptomic analysis of old3-2/oas-a1.1 mutant plants grown under non-stressed conditions suggested the involvement of OAS-A1/OLD3 in plant responses to stress, and in particular, to ROSinduced stress. These findings coincide with phenotypes observed in leaves of old3-2/oas-a1.1 mutants, including histochemical detection of $\mathrm{H}_{2} \mathrm{O}_{2}$ as well as lesions characteristic for cell death [17]. Moreover, a recent research showed the existence of OAS-A1/OLD3 in the peroxisomes, which are actively involved in ROS detoxification in leaves [41]. Thus, the results presented here are consistent with the suggestion that OAS-A1/OLD3 deficiency results in a constitutively reduced capacity to eliminate cytosolic ROS [17]. Moreover the reported phenotype of the oas-a1.1 mutant shows some resemblance with the old3-1 early leaf death phenotype. oas-a1.1 mutant plants have microscopic lesions and the massive cell death observed in old3-1 plants may be the result of the same phenomenon on a much larger scale. The leaf death phenotype coincides with increased expression of the general oxidative stress-related marker DEFL and with other marker genes commonly associated with the activation of the plant defence response. It seems appealing to suggest 
that the presence of odd-ler may amplify the effect of OAS-A1 deficiency on ROS elimination in old3-1 mutant plants. However, the leaf death phenotype requires the presence of the mutated old3-1/OAS-A1 gene, rather than the absence of the wild type one. The question remains how the mutated protein causes the phenotype. It seems inevitable that the Gly ${ }^{162}$ to Glu $^{162}$ substitution causes a conformational change and this may alter the interaction with SAT. It is notable that knockout of four of the 5 SATs causes dwarfism, depending on the SAT remaining [10]. The early leaf death may be an extreme manifestation of the observed dwarfism as a result of an odd-ler-dependent changed interaction between SAT and old3-1. However, an altered conformation could equally well change the function or amplify an otherwise minor role of the protein.

\section{Conclusions}

This study describes an EMS-induced mutation in the cytosolic OAS-TL. The mutation results in a protein that has no in vitro OAS-TL activity anymore, resulting in altered sulphur balance and cadmium hypersensitivity. The mutated gene furthermore causes a temperaturedependent lethal phenotype in combination with the genome-dependent gene odd-ler. The nature of the oddler/old3-1 interaction is still unclear, but the identification of the odd-ler gene, together with analysis of the leaf death syndrome will allow us to unravel this unique cell death pathway.

\section{Methods}

\section{Plant material and growth conditions}

Arabidopsis thaliana accessions Landsberg erecta (Ler-0) and Colombia (Col-0) were mainly used in this study. Several other accessions were obtained from the NASC (Nottingham Arabidopsis Stock Centre). The old3-1 (old3-1old3-1odd-ler odd-ler) mutant has been described elsewhere [24]. old3-1old3-1odd-colodd-col genotype was selected from an $\mathrm{F}_{2}$ population generated from a cross between old3-1OLD3odd-lerodd-ler with Col-0 (OLD3OLD3odd-colodd-col). The old3-1old3-1oddcolodd-col plants therefore carry homozygous Ler-0 DNA around the old3-1 gene and homozygous Col-0 DNA around the odd-col locus, as confirmed with DNA markers. The rest of the genome is mixed Col-0/Ler-0. SALK line 072213, carrying a T-DNA insertion in the OAS-A1/ OLD3 gene in Col-0 background was obtained from NASC under number N572213 [42]. A plant line homozygous for the insertion was designated as old3-2. The T-DNA insertion was detected with a PCR product from the primer pair PrRuG659: TGGTTCACGTAGTGGGCCATCG/PrRuG761: TACACCAATGGAGTGTTCCCAATCA. Surface-sterilized seeds of Ler-0, Col-0, old3-1 and old3-2 genotypes were sown on MS media
[43], $\mathrm{pH}$ 5.6, $1 \%$ sucrose, $1 \%$ agar, and $\mathrm{CdCl}_{2}$ at various concentrations. Seeds were kept at $4^{\circ} \mathrm{C}$ for 3 days and subsequently transferred to a growth chamber $\left(21^{\circ} \mathrm{C}, 16 \mathrm{~h}\right.$ light/ $8 \mathrm{~h}$ dark) for 16 days before harvesting. Soil-grown plants were sown on an organic-rich soil (TULIP PROFI No.4, BOGRO B.V., Hardenberg, The Netherlands) as described [24].

\section{Map-based cloning and plant transformation}

To identify the old3-1 mutation, approximately 5000 homozygous old3-1 (old3-1old3-1odd-lerodd-ler) $\mathrm{F}_{2}$ seedlings with a lethal phenotype were selected from a mapping population generated by crossing heterozygous old3-1 (old3-1OLD3odd-ler odd-ler) plants with Col-0 (OLD3OLD3odd-colodd-col). DNA was isolated using the SHORTY quick preparation method (http:// preuss.bsd.uchicago.edu/protocols/Plantdna.html). The old3-1 mutation was mapped to the Arabidopsis genome using CAPS, SSLP and SNP markers. Potential SNPs [44], were selected and primers that specifically amplified Col0 DNA fragments were designed using the Web SNAPER program [45]. The mutation was mapped onto a $14 \mathrm{~kb}$ region spanning 8 open reading frames. Sequence analyses revealed a single nucleotide change inside $O A S-A 1 /$ OLD3. Oligonucleotide primers PrRuG760 (CTATGATCCTTCCGGTGGTGAGAA) and PrRuG775 (GATGGAAGCAAAGACGCAATGTAACTAA) were used to amplify the mutated $O A S-A 1$ gene from old3-1 plants. The genomic fragment was cloned into pGreen0229 and Agrobacterium-mediated transformation $[46,47]$ of the construct into the wild type Ler-0 was performed to confirm that the old3-1 mutation causes the early leaf death phenotype. In order to distinguish Ho-old3-1 (ie, old31old3-1) and Het-old3-1 (ie, OLD3old3-1) plants, the primers PrRuG760 (CTATGATCCTTCCGGTGGTGAGAA) and PrRuG775 (GATGGAAGCAAAGACGCAATGTAACTAA) were employed to amplify the $O A S$ A1 gene from Ler-0, old3-1old3-1odd-lerodd-ler and old3-1OLD3odd-lerodd-ler plants. The obtained 2928 bp PCR product was digested by Sau96I to visualise the polymorphism between the OLD3 and old3-1 alleles. The odd-ler gene was mapped at the lower arm of chromosome 3, approximately 4 centiMorgans south of SSLP marker K11J14 [24].

\section{Cloning, enzyme activity assay and complementation of $\Delta$ Cys E.coli}

The cytosolic forms of OAS-A1/OLD3 and old3-1 were amplified from Ler-0 and old3-1 cDNAs, respectively, using specific primers as described [28]. The PCR products were sub-cloned into a pGEM-T Easy vector (Promega) and sequenced. The cDNA fragments were cut with NdeI and BamHI and ligated into NcoI/BamHI digested pTrc99A [48] and pET15b (Novagen) to create 
the pTrc99A:OLD3, pTrc99A:old3-1, pET15b:OLD3 and pET15b:old3-1 constructs.

OAS-A1/OLD3 and old3-1 protein was purified from E. coli BL21 (DE3) cells transformed with pET15b:OLD3 and pET15b:old3-1 constructs as described elsewhere [28]. Transformed BL21 (DE3) cells were grown on LB at $37^{\circ} \mathrm{C}$ until $\mathrm{A} 600 \sim 0.8$ and protein expression was induced with $1 \mathrm{mM} 1$-thio- $\beta$-D-galactopyranoside for $4 \mathrm{~h}$ at $37^{\circ} \mathrm{C}$. Bound proteins were eluted from $\mathrm{Ni}^{2+}$-NTA agarose columns with buffer containing $150 \mathrm{mM}$ imidazole. Eluted proteins were dialyzed against $100 \mathrm{mM}$ Mopso (pH 7.0) and stored at $-20^{\circ} \mathrm{C}$. OAS-A1 enzyme activity was determined as described [28] using the Gaitonde method [30]. Briefly, the reaction was started with the addition of 26.7 ng $(3.1 \mathrm{nM})$ of OAS-A1/OLD3 and old3-1 protein and incubated for 5, 10 and $15 \mathrm{~min}$ and subsequently stopped by adding $50 \mu \mathrm{l}$ trichloroacetic acid. After centrifugation, $0.25 \mathrm{ml}$ supernatant was added to $0.25 \mathrm{ml}$ acid-ninhydrin reagent, and placed in a boiling water bath for $5 \mathrm{~min}$.

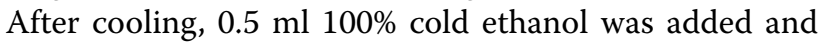
the extinction at $560 \mathrm{~nm}$ was measured. The standard curve for cysteine was made as described [49].

For E. coli NK3 ( $\triangle \operatorname{trpE5}$ leu-6 hsdR hsdM+ cysK cysM) [29] complementation, E. coli cells were transformed with the empty vector pTrc99A, pTrc99A:OLD3 and pTrc99A:old3-1 and grown on 5× M9 Minimal Mediacontaining $1.5 \%$ agar plates, supplemented with $0.2 \mathrm{gL}^{-1}$

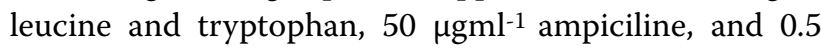
$\mathrm{mM}$ cysteine where indicated.

\section{Physiological analyses}

Chlorophyll content and ion leakage was measured as described [24]. To measure total sulphur, sulphate ion and thiols, plants were grown in soil saturated with $25 \%$ Hoagland solution for 27 days, at $25{ }^{\circ} \mathrm{C}$ and $70 \%$ relative humidity, and a $16 \mathrm{hr} / 8 \mathrm{hr}$ light/dark cycle $\left(200 \mu \mathrm{molm}^{-}\right.$ $\left.{ }^{2} \mathrm{~S}^{-1}\right)$. The above ground parts of plants were harvested for fresh weight and dry weight measurement. Dry plant materials were used for total sulphur measurement using the barium sulphate precipitation method [50] and for anion measurement using HPLC-based methods [51]. Parts of the fresh samples were used for the measurement of total water-soluble non-protein sulfhydryl (SH) compounds following the method described [52]. For the total sulphur and sulphate ion content data of old3-1old31odd-lerodd-ler mutants, one replicate of 50 plants was used for analysis due to the small size of plants and for other data points four replicates of 17 plants were used.

\section{RNA isolation and Real-Time PCR}

Total RNA was isolated and purified using the Qiagen RNeasy plant mini kit according to the manufacturer's protocol. Fifteen hundred nanograms of RNA were used as template for first-strand cDNA synthesis using $200 \mathrm{U}$ of RevertAid $\mathrm{H}$-minus MMuLV reverse transcriptase (Fermentas USA) and an oligo (dT) primer. Primer pairs for Real-Time PCR were designed using the open-source PCR primer design program PerlPrimer v1.1.10 [53]. Applied Biosystems 7300 Real-Time PCR system and the Applied Biosystems power SYBR Green PCR Master Mix kit was used for the Real-Time PCR amplification according to the manufacturer's protocol. Oligonucleotide primers PrRuG2443 (CTTAGTCATTTCCGATGTGCC) and PrRuG2444 (GCATCTTCCACCTTTAGCTC) were used to amplify DEFL (AT2G43510) and primers PrRuG1699 (TCTCCGCTTTGAATTGTCTC) and PrRuG1700 (TATGAGCTTGGAAAGAAAGAGC) were used to amplify ACTIN2 (AT3G18780). The Real-Time PCR program was 2 min at $94^{\circ} \mathrm{C}, 40 \times\left(94^{\circ} \mathrm{C}\right.$ for $10 \mathrm{sec} /$ $58^{\circ} \mathrm{C}$ for $10 \mathrm{sec} / 72^{\circ} \mathrm{C}$ for $\left.25 \mathrm{sec}\right)$. The PCR reaction was followed by a meltcurve analysis to confirm that a single band was amplified and no primer dimers were present. The obtained data was analysed with Bio-Rad software.

\section{Authors' contributions}

RK and HCJ did the physiological and gene expression analysis. HCJ and JS identified the old3-1 mutation. RK did the structure analysis. RK and GE determined the OAS-TL activities. JH participated in the design and coordination of the study. PPD conceived of the study, participated in the design and coordination the work. RK, HCJ and PD drafted the manuscript. All authors contributed to the draft and read and approved the final manuscript.

\section{Acknowledgements}

We would like to thank Dr Jan van der Ploeg from the Swiss Federal Institute of Technology in Zurich for providing the E. coli NK3 strain, Bert Venema and Rene Dekter for technical assistance. Dr Luit de Kok and Bep Stuiver from the University of Groningen for help with the sulphur quantification and Dr Jozeph Jez from the Donald Danforth plant science centre for help with the analysis of the 3D structure of OAS-A1. This work was partially funded by a grant from the Ministry of Science, Research and Technology of Islamic Republic of Iran to Reza Shirzadian- Khorramabad.

\section{Author Details}

1 Molecular Biology of Plants, Groningen Biomolecular Sciences and Biotechnology Institute, University of Groningen, Kerklaan 30, 9751 NN, Haren, The Netherlands, ${ }^{2}$ Centre for Bioenergy Plants Research and Development, Institute of Botany, Chinese Academy of Sciences, Beijing, 100093, China, 3 Institute of Molecular BioSciences (IMBS), Massey University, Private Bag 11222, Palmerston North, New Zealand, ${ }^{4}$ University of Guilan, Department of Agronomy and Plant Breeding, P.O. Box 41635-1314, Rasht, Iran and 5 University of Potsdam, Department of Molecular Biology, Karl-Liebknecht-Str. 24-25, Haus 20, 14476 Potsdam-Golm, Germany

Received: 8 September 2009 Accepted: 29 April 2010 Published: 29 April 2010

\section{References}

1. Wirtz M, Hell R: Dominant-negative modification reveals the regulatory function of the multimeric cysteine synthase protein complex in transgenic tobacco. Plant Cell 2007, 19(2):625-639.

2. May MJ, Vernoux T, Leaver C, Van Montagu M, Inze D: Glutathione homeostasis in plants: implications for environmental sensing and plant development. Journal of Experimental Botany 1998, 49:649-667.

3. Buchner P, Takahashi H, Hawkesford MJ: Plant sulphate transporters: coordination of uptake, intracellular and long-distance transport. J Exp Bot 2004, 55(404):1765-1773.

4. Leustek T, Martin MN, Bick J-A, Davies JP: PATHWAYS AND REGULATION OF SULFUR METABOLISM REVEALED THROUGH MOLECULAR AND GENETIC STUDIES. Annu Rev Plant Physiol Plant Mol Biol 2000, 51:141-165. 
5. Saito K: Regulation of sulfate transport and synthesis of sulfurcontaining amino acids. Curr Opin Plant Biol 2000, 3(3):188-195.

6. Wirtz M, Droux M, Hell R: O-acetylserine (thiol) lyase: an enigmatic enzyme of plant cysteine biosynthesis revisited in Arabidopsis thaliana. J Exp Bot 2004, 55(404):1785-1798.

7. Droux M, Ruffet ML, Douce R, Job D: Interactions between serine acetyltransferase and O-acetylserine (thiol) lyase in higher plants-structural and kinetic properties of the free and bound enzymes. Eur $\lrcorner$ Biochem 1998, 255(1):235-245

8. Feldman-Salit A, Wirtz M, Hell R, Wade RC: A mechanistic model of the cysteine synthase complex. J Mol Biol 2009, 386(1):37-59.

9. Wirtz M, Hell R: Functional analysis of the cysteine synthase protein complex from plants: structural, biochemical and regulatory properties. J Plant Physiol 2006, 163(3):273-286.

10. Watanabe M, Mochida K, Kato T, Tabata S, Yoshimoto N, Noji M, Saito K: Comparative Genomics and Reverse Genetics Analysis Reveal Indispensable Functions of the Serine Acetyltransferase Gene Family in Arabidopsis. Plant Cell 2008, 20(9):2484-2496.

11. Zimmermann $P$, Hirsch-Hoffmann $M$, Hennig L, Gruissem W: GENEVESTIGATOR. Arabidopsis microarray database and analysis toolbox. Plant Physiol 2004, 136(1):2621-2632.

12. Winter $D$, Vinegar $B$, Nahal H, Ammar R, Wilson GV, Provart NJ: An "electronic fluorescent pictograph" browser for exploring and analyzing large-scale biological data sets. PLoS One 2007, 2(1):e718.

13. Hell R, Bork C, Bogdanova N, Frolov I, Hauschild R: Isolation and characterization of two CDNAs encoding for compartment specific isoforms of O-acetylserine (thiol) lyase from Arabidopsis thaliana. FEBS Lett 1994, 351(2):257-262.

14. Hesse H, Lipke J, Altmann T, Hofgen R: Molecular cloning and expression analyses of mitochondrial and plastidic isoforms of cysteine synthase (O-acetylserine(thiol)lyase) from Arabidopsis thaliana. Amino Acids 1999, 16(2):113-131.

15. Hatzfeld Y, Maruyama A, Schmidt A, Noji M, Ishizawa K, Saito K: betaCyanoalanine synthase is a mitochondrial cysteine synthase-like protein in spinach and Arabidopsis. Plant Physiol 2000, 123(3):1163-1171.

16. Yamaguchi $Y$, Nakamura T, Kusano T, Sano H: Three Arabidopsis genes encoding proteins with differential activities for cysteine synthase and beta-cyanoalanine synthase. Plant Cell Physiol 2000, 41(4):465-476.

17. Lopez-Martin MC, Becana M, Romero LC, Gotor C: Knocking out cytosolic cysteine synthesis compromises the antioxidant capacity of the cytosol to maintain discrete concentrations of hydrogen peroxide in Arabidopsis. Plant Physiol 2008, 147(2):562-572.

18. Heeg C, Kruse C, Jost R, Gutensohn M, Ruppert T, Wirtz M, Hell R: Analysis of the Arabidopsis O-acetylserine(thiol)lyase gene family demonstrates compartment-specific differences in the regulation of cysteine synthesis. Plant Cell 2008, 20(1):168-185.

19. Noctor G, Strohm M, Jouanin L, Kunert KJ, Foyer CH, Rennenberg H: Synthesis of Glutathione in Leaves of Transgenic Poplar Overexpressing [gamma]-Glutamylcysteine Synthetase. Plant Physiol 1996, 112(3):1071-1078

20. Strohm M, Jouanin L, Kunert KJ, Pruvost C, Polle A, Foyer CH, Rennenberg H: REGULATION OF GLUTATHIONE SYNTHESIS IN LEAVES OF TRANSGENIC POPLAR (POPULUS-TREMULA X POPULUS-ALBA) OVEREXPRESSING GLUTATHIONE SYNTHETASE. Plant Journal 1995, 7(1):141-145

21. Cobbett CS: Phytochelatins and their roles in heavy metal detoxification. Plant Physiol 2000, 123(3):825-832.

22. Meyer AJ, Hell R: Glutathione homeostasis and redox-regulation by sulfhydryl groups. Photosynth Res 2005, 86(3):435-457.

23. Mullineaux PM, Rausch T: Glutathione, photosynthesis and the redox regulation of stress-responsive gene expression. Photosynth Res 2005, 86(3):459-474

24. Jing H-C, Sturre MJG, Hille J, Dijkwel PP: Arabidopsis onset of leaf death mutants identify a regulatory pathway controlling leaf senescence. Plant J 2002, 32(1):51-63.

25. Brodersen P, Petersen M, Pike HM, Olszak B, Skov S, Ødum N, Jørgensen LB, Brown RE, Mundy J: Knockout of Arabidopsis ACCELERATED-CELLDEATH11 encoding a sphingosine transfer protein causes activation of programmed cell death and defense. Genes \& Development 2002, 16(4):490-502.
26. Gadjev I, Vanderauwera S, Gechev TS, Laloi C, Minkov IN, Shulaev V, Apel K, Inze D, Mittler R, Van Breusegem F: Transcriptomic footprints disclose specificity of reactive oxygen species signaling in Arabidopsis. Plant Physio/ 2006, 141(2):436-445.

27. Bogdanova N, Hell R: Cysteine synthesis in plants: protein-protein interactions of serine acetyltransferase from Arabidopsis thaliana. Plant J 1997, 11(2):251-262.

28. Bonner ER, Cahoon RE, Knapke SM, Jez JM: Molecular basis of cysteine biosynthesis in plants: structural and functional analysis of Oacetylserine sulfhydrylase from Arabidopsis thaliana. J Biol Chem 2005, 280(46):38803-38813.

29. Ploeg JR van der, Barone M, Leisinger T: Functional analysis of the Bacillus subtilis cysK and cysJ genes. FEMS Microbiol Lett 2001, 201(1):29-35.

30. Gaitonde MK: A spectrophotometric method for the direct determination of cysteine in the presence of other naturally occurring amino acids. Biochem J 1967, 104(2):627-633.

31. Burandt P, Schmidt A, Papenbrock J: Three O -acetyl-L-serine(thiol)lyase isoenzymes from Arabidopsis catalyse cysteine synthesis and cysteine desulfuration at different $\mathrm{pH}$ values. Journal of Plant Physiology 2002, 159(2):111-119.

32. Jost R, Berkowitz O, Wirtz M, Hopkins L, Hawkesford MJ, Hell R: Genomic and functional characterization of the oas gene family encoding $\mathrm{O}$ acetylserine (thiol) lyases, enzymes catalyzing the final step in cysteine biosynthesis in Arabidopsis thaliana. Gene 2000, 253(2):237-247.

33. Hesse H, Nikiforova V, Gakiere B, Hoefgen R: Molecular analysis and control of cysteine biosynthesis: integration of nitrogen and sulphur metabolism. Journal of Experimental Botany 2004, 55(401):1283-1292.

34. Howarth JR, Dominguez-Solis JR, Gutierrez-Alcala G, Wray JL, Romero LC, Gotor C: The serine acetyltransferase gene family in Arabidopsis thaliana and the regulation of its expression by cadmium. Plant Mol Biol 2003, 51(4):589-598.

35. Rauser WE: Metal-Binding Peptides in Plants. Sulfur Nutrition and Assimilation in Higher Plants 1993:239-251.

36. Dominguez-Solis JR, Lopez-Martin MC, Ager FJ, Ynsa MD, Romero LC, Gotor C: Increased cysteine availability is essential for cadmium tolerance and accumulation in Arabidopsis thaliana. Plant Biotechnol J 2004, 2(6):469-476.

37. Francois JA, Kumaran S, Jez JM: Structural basis for interaction of Oacetylserine sulfhydrylase and serine acetyltransferase in the Arabidopsis cysteine synthase complex. Plant Cell 2006, 18(12):3647-3655

38. Barth $\mathrm{S}$, Melchinger AE, Lubberstedt T: Genetic diversity in Arabidopsis thaliana L. Heynh. investigated by cleaved amplified polymorphic sequence (CAPS) and inter-simple sequence repeat (ISSR) markers. Mol Ecol 2002, 11(3):495-505.

39. Dominguez-Solis JR, Gutierrez-Alcala G, Vega JM, Romero LC, Gotor C: The cytosolic O-acetylserine(thiol)lyase gene is regulated by heavy metals and can function in cadmium tolerance. J Biol Chem 2001, 276(12):9297-9302.

40. Barroso C, Romero LC, Cejudo FJ, Vega JM, Gotor C: Salt-specific regulation of the cytosolic $\mathrm{O}$-acetylserine(thiol)lyase gene from Arabidopsis thaliana is dependent on abscisic acid. Plant Mol Biol 1999, 40(4):729-736.

41. Reumann S, Babujee L, Ma C, Wienkoop S, Siemsen T, Antonicelli GE, Rasche N, Luder F, Weckwerth W, Jahn O: Proteome analysis of Arabidopsis leaf peroxisomes reveals novel targeting peptides, metabolic pathways, and defense mechanisms. Plant Cell 2007, 19(10):3170-3193.

42. Alonso JM, Stepanova AN, Leisse TJ, Kim CJ, Chen H, Shinn P, Stevenson DK, Zimmerman J, Barajas P, Cheuk R, et al: Genome-wide insertional mutagenesis of Arabidopsis thaliana. Science 2003, 301(5633):653-657.

43. Murashige T, Skoog F: A Revised Medium for Rapid Growth and Bio Assays with Tobacco Tissue Cultures. Physiologia Plantarum 1962, 15(3):473-497.

44. Jander G, Norris SR, Rounsley SD, Bush DF, Levin IM, Last RL: Arabidopsis map-based cloning in the post-genome era. Plant Physiol 2002, 129(2):440-450

45. Drenkard E, Richter BG, Rozen S, Stutius LM, Angell NA, Mindrinos M, Cho RJ, Oefner PJ, Davis RW, Ausubel FM: A simple procedure for the analysis of single nucleotide polymorphisms facilitates map-based cloning in Arabidopsis. Plant Physiol 2000, 124(4):1483-1492. 
46. Hellens RP, Edwards EA, Leyland NR, Bean S, Mullineaux PM: pGreen: a versatile and flexible binary Ti vector for Agrobacterium-mediated plant transformation. Plant Mol Biol 2000, 42(6):819-832.

47. Clough SJ, Bent AF: Floral dip: a simplified method for Agrobacteriummediated transformation of Arabidopsis thaliana. Plant J 1998, 16(6):735-743.

48. Amann $\mathrm{E}, \mathrm{Ochs} \mathrm{B}$, Abel $\mathrm{KJ}$ : Tightly regulated tac promoter vectors useful for the expression of unfused and fused proteins in Escherichia coli. Gene 1988, 69(2):301-315.

49. Nakamura K, Hayama A, Masada M, Fukushima K, Tamura G: Measurement Of Serine Acetyltransferase Activity in Crude PlantExtracts By a Coupled Assay System Using Cysteine Synthase. Plant and Cell Physiology 1987, 28(5):885-891.

50. Jones JB Jr: Determining total sulphur in plant tissue using the $\mathrm{HACH}$ kit spectrophotometer technique. Sulphur in Agriculture 1995, 19:58-62.

51. Maas FM, Hoffmann I, Vanharmelen MJ, Dekok LJ: Refractometric Determination Of Sulfate And Other Anions in Plants Separated By High-Performance Liquid-Chromatography. Plant and Soil 1986, 91(1):129-132.

52. Dekok LJ, Graham M: Levels Of Pigments, Soluble-Proteins, AminoAcids And Sulfhydryl Compounds in Foliar Tissue Of ArabidopsisThaliana During Dark-Induced And Natural Senescence. Plant Physiology and Biochemistry 1989, 27(2):203-209.

53. Marshall OJ: PerlPrimer: cross-platform, graphical primer design for standard, bisulphite and real-time PCR. Bioinformatics 2004, 20(15):2471-2472

doi: 10.1186/1471-2229-10-80

Cite this article as: Shirzadian-Khorramabad et al., A mutation in the cytosolic O-acetylserine (thiol) lyase induces a genome-dependent early leaf death phenotype in Arabidopsis BMC Plant Biology 2010, 10:80

Submit your next manuscript to BioMed Central and take full advantage of:

- Convenient online submission

- Thorough peer review

- No space constraints or color figure charges

- Immediate publication on acceptance

- Inclusion in PubMed, CAS, Scopus and Google Scholar

- Research which is freely available for redistribution

Submit your manuscript at www.biomedcentral.com/submit
Ciomed Central 